\title{
GLOBAL COMPARISONS OF MEDIANS AND OTHER QUANTILES IN A ONE-WAY DESIGN WHEN THERE ARE TIED VALUES
}

\author{
Rand R. Wilcox \\ Dept of Psychology \\ University of Southern California
}

June 25, 2015 


\begin{abstract}
For $J \geq 2$ independent groups, the paper deals with testing the global hypothesis that all $J$ groups have a common population median or identical quantiles, with an emphasis on the quartiles. Classic rank-based methods are sometimes suggested for comparing medians, but it is well known that under general conditions they do not adequately address this goal. Extant methods based on the usual sample median are unsatisfactory when there are tied values except for the special case $J=2$. A variation of the percentile bootstrap used in conjunction with the Harrell-Davis quantile estimator performs well in simulations. The method is illustrated with data from the Well Elderly 2 study.
\end{abstract}

Keywords: Tied values; bootstrap methods; Harrell-Davis estimator; projection distances; Well Elderly 2 study

\title{
1 Introduction
}

For $J$ independent random variables, let $\theta_{j}$ be the population median or some other quantile associated with $j$ th variable $(j=1, \ldots, J)$. The paper considers the problem of testing

$$
H_{0}: \theta_{1}=\cdots=\theta_{J}
$$

particularly when there are tied values. The focus is on comparing the medians as well as the upper or lower quartiles, but it is evident that the results are relevant when comparing other quantiles instead.

For the special case $J=2$, the Wilcoxon-Mann-Whitney (WMW) test is sometimes suggested for comparing medians, but it is well known that under general conditions it does not accomplish this goal (e.g., Hettmansperger, 1984; Fung, 1980). Roughly, the reason is that for two independent random variables, $X$ and $Y$, it is not based on an estimate of $\theta_{1}-\theta_{2}$, but rather on an estimate of $P(X<Y)$. Another concern is that when distributions differ, under general conditions the WMW test uses the wrong standard error (e.g., Cliff, 1996; Wilcox, 2012). More generally, the Kruskall-Wallis test, which reduces to the WilcoxonMann-Whitney test when $J=2$, does not test (1). 
Yet another possible approach is to use the usual sample median in conjunction with a permutation test. However, results in Romano (1990) establish that this approach is unsatisfactory as well.

For a random sample $X_{1}, \ldots, X_{n}$, let $X_{(1)} \leq \ldots \leq X_{(n)}$ denote the observations written in ascending order and let $M_{j}$ denote the usual sample median. That is, if the number of observations, $n$, is odd,

$$
M=X_{(m)}
$$

where $m=(n+1) / 2$ and if $n$ is even,

$$
M=\frac{\left(X_{(m)}+X_{(m+1)}\right)}{2},
$$

where now $m=n / 2$. A natural way of proceeding is to estimate $\theta_{j}$ with $M_{j}$ and use some test statistic that is based in part on some estimate of the standard error of $M_{j}$. When sampling from a continuous distribution where tied values occur with probability zero, an effective method was studied by Bonett and Price (2002) that can be used when $J=2$ or when $J>2$ and the goal is to test some hypothesis based on a linear contrast of the population medians. Numerous methods for estimating the standard error of $M_{j}$ have been derived, but extant results indicate that all of them can perform poorly when tied values can occur (Wilcox, 2012). Wilcox (2006) found a slight extension of a standard percentile bootstrap method that performs well when testing (1), there are tied values and $J=2$. But Wilcox (2012) notes that in terms of testing (1) when $J>2$, evidently no method has been found that performs well in simulations when there are tied values.

There is another complication when working with the usual sample median. It is well known that when sampling from a continuous distribution, under certain regularity conditions, $M_{j}$ is asymptotically normal. However, when sampling from a discrete distribution with a finite sample space, $M_{j}$ does not converge to a normal distribution. More broadly, when estimating quantiles using a single order statistic, or a weighted average of two order statistics, assuming asymptotic normality is generally unsatisfactory when dealing with discrete distributions where tied values occur.

As an illustration, consider a beta-binomial distribution having the probability function

$$
P(x)=\frac{\mathbf{B}(m-x+r, x+s)}{(m+1) \mathbf{B}(m-x+1, x+1) \mathbf{B}(r, s)},
$$


where $\mathbf{B}$ is the complete beta function, $r>0$ and $s>0$ are parameters that determine the shape of the distribution and $x=0, \ldots, m$. Consider $m=30$. Then the cardinality of the sample space is 31 and as is evident, if the sample size is $n>31$, tied values occur with probability one. The left panel of Figure 1 shows a plot of 3000 sample medians generated from a beta-binomial distribution with $r=1$ and $s=3$ based on a sample size $n=100$. (So the beta-binomial distribution is skewed to the right, $P(x)$ is monotonic decreasing and $x=6$ corresponds to the .52 quantile.) The right panel is the same as the left, only now $n=500$. As is evident, the sampling distribution has not moved closer to a normal distribution and indeed the cardinality of the sample space has decreased, indicating that any method for making inferences based on the sample median that assumes asymptotic normality can perform poorly.

For the special case where the goal is to compare two independent groups, a method for comparing quantiles that deals effectively with tied values is to use a percentile bootstrap in conjunction with the quantile estimator derived by Harrell and Davis (1982); see Wilcox et al. (2013). The Harrell-Davis estimator uses a weighted average of all the order statistics. The result is a sampling distribution that is typically well approximated by a continuous distribution. Consider again the right panel of Figure 1 and note that the cardinality of the sample space is only five. That is, only five values for the sample median were observed among the 3000 estimates. In contrast, if the Harrell-Davis estimator is used, there are no tied values among all 3000 estimates. But the method studied by Wilcox et al. is limited to $J=2$; there are no results on how best to proceed when testing (1) and there are $J>2$ independent groups.

Here, two methods for testing (1) were considered, both of which were based on the Harrell-Davis estimator. The first was based on a test statistic mentioned by Schrader and Hettmansperger (1980), and studied by He, Simpson and Portnoy (1990). The basic strategy was to use a percentile bootstrap method to estimate the null distribution. But situations were found where this approach performed very poorly when dealing with tied values, so further details are omitted. The other method is described in section 2 and simulation results are reported in section 3. Section 4 illustrates the proposed method using data from the Well Elderly 2 study. The strategy is not new and has been found to perform reasonably well when dealing with other robust measures of location (Wilcox, 2012). However, when 

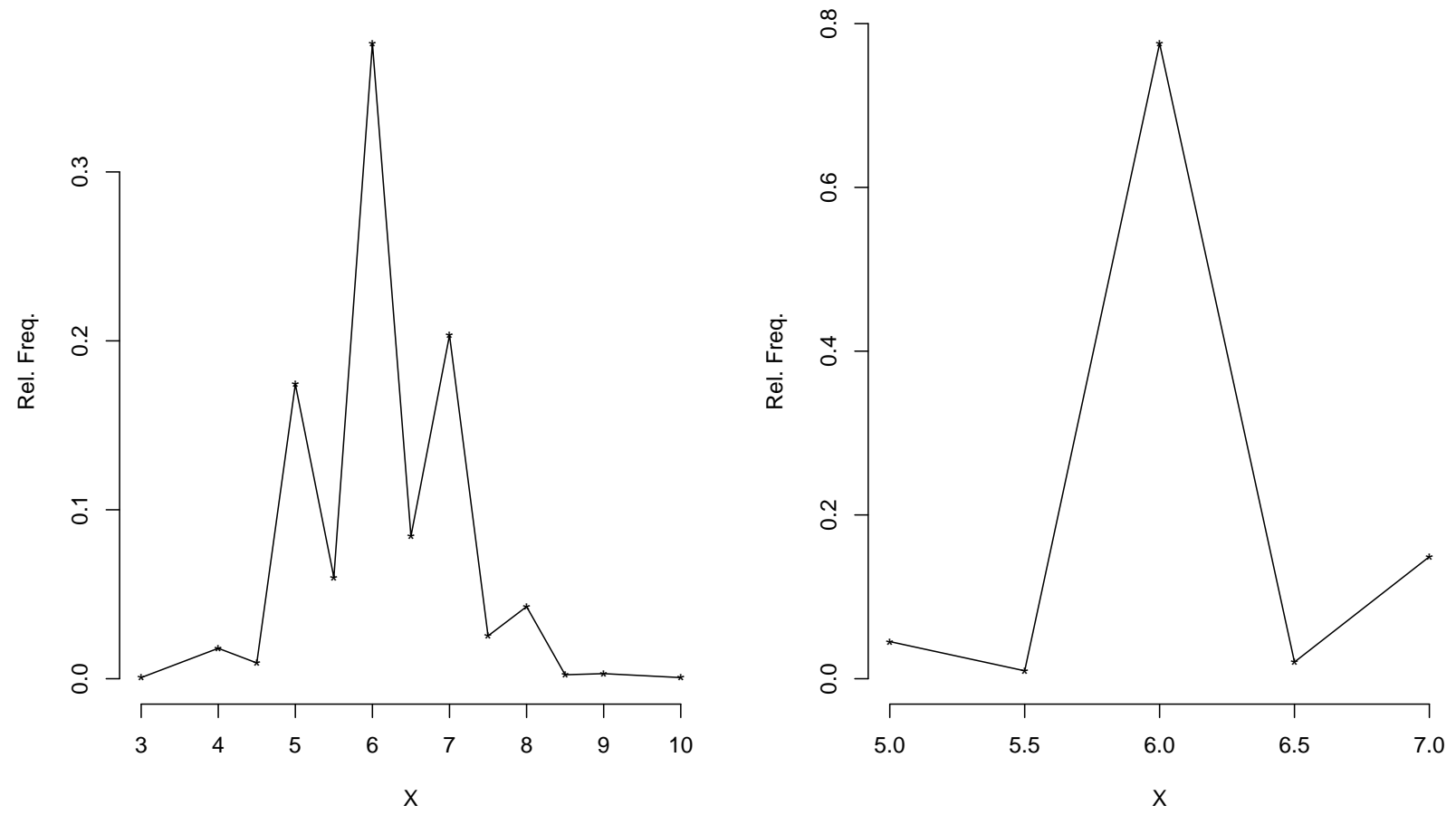

Figure 1: The left panel shows a plot of 3000 sample medians when sampling from a betabinomial distribution, $n=100, m=30, r=1, s=3$. The right panel is a plot of the 3000 medians when $n=500$ 
using the usual sample median for the situation at hand, preliminary simulations found that it performs poorly in terms of controlling the Type I error probability. Results in Wilcox et al. (2013) suggest that using instead the Harrell-Davis estimator, reasonably good control over the Type I error probability might be obtained. So the goal here is to determine the extent to which this is the case.

Note that in terms of characterizing the typical value of some random variable, the population median is an obvious choice. However, situations are encountered where differences occur in the tails of a distribution that have substantive interest (e.g. Doksum \& Sievers, 1976; Wilcox et al., 2014). This issue can be addressed by comparing quantiles other than the median, which can help provide a deeper understanding of how distributions differ. This point is illustrated in section 4 .

\section{Description of the Proposed Method}

To describe the Harrell and Davis (1982) estimate of the qth quantile, let $Y$ be a random variable having a beta distribution with parameters $a=(n+1) q$ and $b=(n+1)(1-q)$. That is, the probability density function of $Y$ is

$$
\frac{\Gamma(a+b)}{\Gamma(a) \Gamma(b)} y^{a-1}(1-y)^{b-1}, 0 \leq y \leq 1
$$

where $\Gamma$ is the gamma function. Let

$$
W_{i}=P\left(\frac{i-1}{n} \leq Y \leq \frac{i}{n}\right)
$$

For a random sample $X_{1}, \ldots, X_{n}$, let $X_{(1)} \leq \ldots \leq X_{(n)}$ denote the observations written in ascending order. The Harrell-Davis estimate of $\theta_{q}$, the $q$ th quantile, is

$$
\hat{\theta}_{q}=\sum_{i=1}^{n} W_{i} X_{(i)}
$$

In terms of its standard error, Sfakianakis and Verginis (2006) show that in some situations the Harrell-Davis estimator competes well with alternative estimators that again use a weighted average of all the order statistics, but there are exceptions. (Sfakianakis and Verginis derived alternative estimators that have advantages over the Harrell-Davis in some 
situations. But when sampling from heavy-tailed distributions, the standard errors of their estimators can be substantially larger than the standard error of $\hat{\theta}_{q}$.) Additional comparisons of various estimators are reported by Parrish (1990), Sheather and Marron (1990), as well as Dielman, Lowry and Pfaffenberger (1994). The only certainty is that no single estimator dominates in terms of efficiency. For example, the Harrell-Davis estimator has a smaller standard error than the usual sample median when sampling from a normal distribution or a distribution that has relatively light tails, but for sufficiently heavy-tailed distributions, the reverse is true (Wilcox, 2012, p. 87).

Let $\delta_{j k}=\theta_{j}-\theta_{k}$. Roughly, the strategy for testing (1) is to test

$$
H_{0}: \delta_{j k}=0, \forall j<k
$$

via a percentile bootstrap method. If the null hypothesis is true, then $\mathbf{0}$, a vector of zeros having length $\left(J^{2}-J\right) / 2$, should be nested fairly deeply within a cloud of bootstrap estimates of $\delta_{j k}$. Moreover, the depth of $\mathbf{0}$ can be used to compute a p-value as will be indicated. A natural way of measuring the depth of $\mathbf{0}$ within a bootstrap cloud is via Mahalanobis distance. Note, however, that the $\delta_{j k}$ parameters are linearly dependent. This indicates that Mahalanobis distance can fail from a computational point of view because the bootstrap covariance matrix will be singular. This proved to be the case, so the strategy here is to measure the depth of $\mathbf{0}$ using a method that does not require the use of a covariance matrix.

For completeness, note that the issue of a singular covariance matrix could be avoided by using the first group as a reference group and testing $H_{0}: \theta_{1}-\theta_{2}=\theta_{1}-\theta_{3}=\cdots=\theta_{1}-\theta_{J}$. In terms of a Type I error, this approach is reasonable, but in terms of power, this is not necessarily the case. The reason is that power can depend on which group is used as the reference group because the choice of the reference group impacts the magnitude of the differences between the medians that are compared.

To describe the details of the proposed test of (1) via (4), let $X_{i j}$ be a random sample from the $j$ th group $\left(i=1, \ldots, n_{j}\right)$. Generate a bootstrap sample from the $j$ th group by resampling with replacement $n_{j}$ observations from group $j$. Let $\hat{\theta}_{j}^{*}$ be the estimate of the $q$ th quantile for group $j$ based on this bootstrap sample. Let $\hat{\delta}_{j k}^{*}=\hat{\theta}_{j}^{*}-\hat{\theta}_{k}^{*}, j<k$. Repeat this process $B$ times yielding $\hat{\delta}_{b j k}^{*}, b=1, \ldots, B$. Here, $B=600$ is used in order to avoid overly high execution time and because this choice has been found to provide reasonably good 
control over the Type I error probability when dealing with related bootstrap techniques (e.g., Wilcox, 2012). However, in terms of power, there might be a practical advantage to using a larger choice for $B$ (Racine \& MacKinnon, 2007).

A portion of the strategy used here is based on measuring the depth of a point in a multivariate data cloud using a projection-type method, which provides an approximation of half-space depth (Wilcox, 2012, section 6.2.5). For notational convenience, momentarily focus on an $n \times p$ matrix of data, $\mathbf{Y}$. Let $\hat{\tau}$ be some measure of location based on $\mathbf{Y}$. For simplicity, the marginal medians (based on the usual sample median) are used. Let

$$
\mathbf{U}_{i}=\mathbf{Y}_{i}-\hat{\tau}
$$

$(i=1, \ldots, n)$

$$
C_{i}=\mathbf{U}_{i} \mathbf{U}_{i}^{\prime}
$$

and for any $j(j=1, \ldots, n)$, let

$$
\begin{gathered}
W_{i j}=\sum_{k=1}^{J} U_{i k} U_{j k}, \\
T_{i j}=\frac{W_{i j}}{C_{i}}\left(U_{i 1}, \ldots, U_{i p}\right)
\end{gathered}
$$

and

$$
D_{i j}=\left\|T_{i j}\right\|,
$$

where $\left\|T_{i j}\right\|$ is the Euclidean norm associated with the vector $T_{i j}(i=1, \ldots n ; j=1, \ldots, n)$. Let

$$
d_{i j}=\frac{D_{i j}}{q_{i 2}-q_{i 1}}
$$

where $q_{i 2}$ and $q_{i 1}$ are estimates of the upper and lower quartiles, respectively, based on $D_{i 1}, \ldots, D_{i n}$. Here, $q_{i 2}$ and $q_{i 1}$ are estimated with the so-called ideal fourths (e.g., Friqqe et al., 1989.), which are computed as follows. Let $j$ be the integer portion of $(n / 4)+(5 / 12)$ and let

$$
h=\frac{n}{4}+\frac{5}{12}-j
$$

The lower quartile is estimated with $\mathrm{s}$

$$
q_{i 1}=(1-h) D_{i(j)}+h D_{i(j+1)},
$$


where $D_{i(1)} \leq \cdots \leq D_{i(n)}$. Letting $k=n-j+1$, the upper quartile is

$$
q_{i 2}=(1-h) D_{i(k)}+h D_{i(k-1)} .
$$

The projection distance of $\mathbf{Y}_{j}$, relative to the cloud of points represented by $\mathbf{Y}$, is the maximum value of $d_{i j}$, the maximum being taken over $i=1, \ldots, n$. This measure of depth is nearly the same as the measure derived by Donoho and Gasko (1992). the only difference is that they used the median absolute difference (mad) as a measure of scale rather than the interquartiles range. MAD has a higher breakdown point but using the interquartile range has been found to perform better in various situations (Wilcox, 2012). This might be due to the poor efficiency of mad, but the extent this is the case is unclear. Perhaps using mad would perform well in the simulations reported here, but this is left to future investigations.

Now create a $(B+1) \times L$ matrix $\mathbf{G}$ where the first $B$ rows are based on the $\hat{\delta}_{b j k}^{*}$, $b=1, \ldots, B, L=\left(J^{2}-J\right) / 2$. That is, row $b$ consists of the $L$ values associated with $\hat{\delta}_{b j k}^{*}$ for all $j<k$. Row $B+1$ of $\mathbf{G}$ is a vector $\mathbf{0}$ having length $L$. Then from general theoretical results in Liu and Singh (1997), a (generalized) p-value can be computed based on the relative distance of $\mathbf{0}$. Compute the projection distance for each row of $\mathbf{G}$. The distance associated with the $b$ th row is denoted by $K_{b}$ and the distance for the null vector (row $B+1$ ) is denoted by $K_{0}$. Then a generalized p-value is

$$
1-\frac{1}{B} \sum_{b=1}^{B} I\left(K_{0} \geq K_{b}\right)
$$

where the indicator function $I\left(K_{0} \geq K_{b}\right)=1$ if $K_{0} \geq K_{b}$, otherwise $I\left(K_{0} \geq K_{b}\right)=0$. This will be called method Q.

\section{Simulation Results}

Simulations were used to study the small-sample properties of method Q when there are $J=4$ groups. Results are reported when comparing medians as well as the lower and upper quartiles. Estimated Type I error probabilities, $\hat{\alpha}$, were based on 4000 replications. Both continuous and discrete distributions were used. The four continuous distributions were normal, symmetric and heavy-tailed, asymmetric and light-tailed, and asymmetric and 
Table 1: Some properties of the g-and-h distribution.

\begin{tabular}{ccrr}
\hline $\mathrm{g}$ & $\mathrm{h}$ & $\kappa_{1}$ & $\kappa_{2}$ \\
\hline 0.0 & 0.0 & 0.00 & 3.0 \\
0.0 & 0.2 & 0.00 & 21.46 \\
0.2 & 0.0 & 0.61 & 3.68 \\
0.2 & 0.2 & 2.81 & 155.98 \\
\hline
\end{tabular}

heavy-tailed. More precisely, four g-and-h distributions were used (Hoaglin, 1985) that contain the standard normal distribution as a special case. If $Z$ has a standard normal distribution, then

$$
W= \begin{cases}\frac{\exp (g Z)-1}{g} \exp \left(h Z^{2} / 2\right), & \text { if } g>0 \\ Z \exp \left(h Z^{2} / 2\right), & \text { if } g=0\end{cases}
$$

has a g-and-h distribution where $g$ and $h$ are parameters that determine the first four moments. The four distributions used here were the standard normal $(g=h=0.0)$, a symmetric heavy-tailed distribution $(h=0.2, g=0.0)$, an asymmetric distribution with relatively light tails $(h=0.0, g=0.2)$, and an asymmetric distribution with heavy tails $(g=h=0.2)$. Table 1 shows the skewness $\left(\kappa_{1}\right)$ and kurtosis $\left(\kappa_{2}\right)$ for each distribution. Additional properties of the g-and-h distribution are summarized by Hoaglin (1985).

As for situations where tied values can occur, consider a discrete distribution with a sample space having cardinality $N$. A goal in the simulations was to get some sense about how well method Q controls the Type I error probability when $N$ is small. Roughly, as the likelihood of tied values increases, at what point does method Q break down? Here, results are reported when data are generated from a beta-binomial distribution for which the cardinality of the sample space is $N=m+1=11$ and $N=m+1=21$. The choices for $(r, s)$ were $(3,3)$, which has a symmetric distribution, as well as $(1,3)$ and $(1,9)$, which are skewed distributions.

First consider the four g-and-h distributions when testing at the .05 level and the groups have a common sample size $n=20$. As indicated in Table 2, the estimated Type I error probability ranges between .025 and .062. Although the importance of a Type I error depends on the situation, Bradley (1978) suggests that as a general guide, when testing at the .05 level, the actual level should not drop below .025 or exceed .075. In Table 2, the estimates 
Table 2: Estimated Type I Error Probability using method Q, continuous distributions, $\alpha=.05$

\begin{tabular}{ccccc}
$q$ & $g$ & $h$ & $n=20$ & $n=50$ \\
\hline 0.25 & 0.0 & 0.0 & 0.058 & 0.059 \\
0.25 & 0.0 & 0.2 & 0.031 & 0.046 \\
0.25 & 0.2 & 0.0 & 0.061 & 0.058 \\
0.25 & 0.2 & 0.2 & 0.038 & 0.055 \\
0.50 & 0.0 & 0.0 & 0.059 & 0.061 \\
0.50 & 0.0 & 0.2 & 0.046 & 0.057 \\
0.50 & 0.2 & 0.0 & 0.062 & 0.062 \\
0.50 & 0.2 & 0.2 & 0.054 & 0.056 \\
0.75 & 0.2 & 0.0 & 0.049 & 0.054 \\
0.75 & 0.2 & 0.2 & 0.025 & 0.038 \\
\hline
\end{tabular}

were in this range.

A possible criticism of the results in Table 2 is that they are based on only 4000 replications. Consequently, some comments about the precision of the estimates in Table 2 are provided. Assuming Bradley's criterion is reasonable, consider the issue of whether the actual level is less than or equal .075. Using the method in Pratt (1968), it can be seen that based on a two-sided .95 confidence interval for the actual level, the confidence interval will not contain .075 if $\hat{\alpha} \leq .06675$. All of the results in Table 2 suggest that the actual level does not exceed .075. Using instead a .99 confidence interval for the actual level, $\hat{\alpha} \leq .06425$ indicates that the actual level does not exceed .075. In a similar manner, based on a twosided .95 confidence interval, the confidence interval for the actual level does not contain .025 if $\hat{\alpha} \geq .03025$. For a .99 confidence interval, $\hat{\alpha} \geq .032$ is required and there are only two situations where the estimate is less than .032 which occurred for $n=20$ when comparing the quartiles.

For normal distributions, a simulation was run with $n=100$ as an additional check on how the method performs as $n$ gets large. The estimated Type I error probability was .058.

For the beta-binomial distributions, estimated Type I error probabilities are shown in Table 3. For $m=20$, control over the Type I error probability is reasonably good when 
Table 3: Estimated probability of a Type I error using method Q when sampling from a betabinomial distribution for a sample sizes $n=20$ and 50, $\alpha=.05$, and where the cardinality of the sample space is $N=m+1$.

\begin{tabular}{ccccccc}
$q$ & $r$ & $s$ & $(n, m)=(20,10)$ & $(n, m)=(20,20)$ & $(n, m)=(50,10)$ & $(n, m)=(50,20)$ \\
\hline 0.25 & 3 & 3 & 0.074 & 0.071 & 0.068 & 0.063 \\
0.50 & 3 & 3 & 0.066 & 0.070 & 0.061 & 0.058 \\
0.25 & 1 & 3 & 0.056 & 0.052 & 0.094 & 0.048 \\
0.50 & 1 & 3 & 0.059 & 0.062 & 0.060 & 0.060 \\
0.75 & 1 & 3 & 0.078 & 0.085 & 0.065 & 0.067 \\
0.25 & 1 & 9 & 0.008 & 0.058 & 0.000 & 0.067 \\
0.50 & 1 & 9 & 0.088 & 0.052 & 0.154 & 0.050 \\
0.75 & 1 & 9 & 0.061 & 0.064 & 0.069 & 0.054 \\
\hline
\end{tabular}

comparing medians. But for $m=10$, it is evident that control over the probability of a Type can be unsatisfactory, particularly when $(r, s)=(1,9)$. The fact that method $\mathrm{Q}$ does not perform well for this particular distribution is not surprising because the .47 quantile is zero. When comparing the quartiles with $m=20$, method Q can be unsatisfactory with $n=20$, the highest estimate of actual level being .085. For this particular situation, increasing the sample size of two of the groups to 40, the estimate is .064. With all sample sizes equal to 30 and $m=10$, the estimate is .065 .

Precise details regarding the rate of convergence to the nominal level is impossible with only 4000 replications, but it is evident that the rate of convergence can depend on the nature of the distribution. Among the discrete distributions considered here for which the cardinality of the sample space is $21, n \geq 20$ suffices in terms of achieving an estimated Type I error probability reasonably close to a nominal .05 level when comparing the medians. But for $m=10$ (the cardinality of the sample space is 11 ) and $(r, s)=(1,9), n \geq 180$ is required when comparing medians. With $n=100$, for example, the estimate of the actual level exceeds .09, in which case the .95 confidence interval for the actual level does not contain .075 .

A few simulations were performed using a discrete distribution where the null hypothesis is true but not all of the distributions are identical. All indications are that when comparing 
medians, again the Type I error probability is controlled reasonably well when $n=m=20$. Consider, for example, a beta-binomial distribution where $r=s=3$. Then 11 is the .54 quantile. Now, suppose that for all four groups data are generated from a discrete distribution such that $P(X \leq x)$ corresponds to a beta-binomial distribution where $r=s=3$ provided that $x \leq 11$, but that otherwise some of the cumulative distributions differ. So the distributions are not all identical in the right tail, but the hypothesis of equal population medians is true. Consider in particular the situation where for three of the groups the probability function, say $f(x)$, corresponds to a beta-binomial probability function when $x<15$. Otherwise

$$
f(x)=\sum_{x=15}^{21} P(x) / 7
$$

when $x \geq 15$, where again $P(x)$ indicates a a beta-binomial distribution given by (2). Now the estimated Type I error probability when comparing the population medians is .057. If instead for $x=15,16, \ldots, 21 f(x)$ is taken to be $P(21), P(20), \ldots, P(15)$, respectively, the estimated Type I error probability is .048. However, when comparing the lower quartiles, now control over the Type I error probability exceeds .09. Increasing the sample sizes to 40, this problem persisted. With all sample sizes equal to 50, control over the Type I error probability is reasonably good, the estimate being .064 .

\section{An Illustration}

Method Q is illustrated using data from the Well Elderly 2 study (Jackson, et al., 2009; Clark et al. 1997). Generally, the study dealt with the efficacy of a particular intervention strategy aimed at improving the physical and emotional health of older adults. One particular issue was whether four educational groups differed in terms of a measure of meaningful activity prior to intervention. The four groups were high school graduate, some college or technical school, 4 years of college and post-graduate school. For convenience, these groups are designated G1, G2, G3 and G4 henceforth. Meaningful activity was measured with the sum of 29 Likert items, where the possible responses for each item were $0,1,2,3$ and 4. Higher scores reflect higher levels of meaningful activities. The sample sizes were 62, 81, 110 and 125 , respectively. 


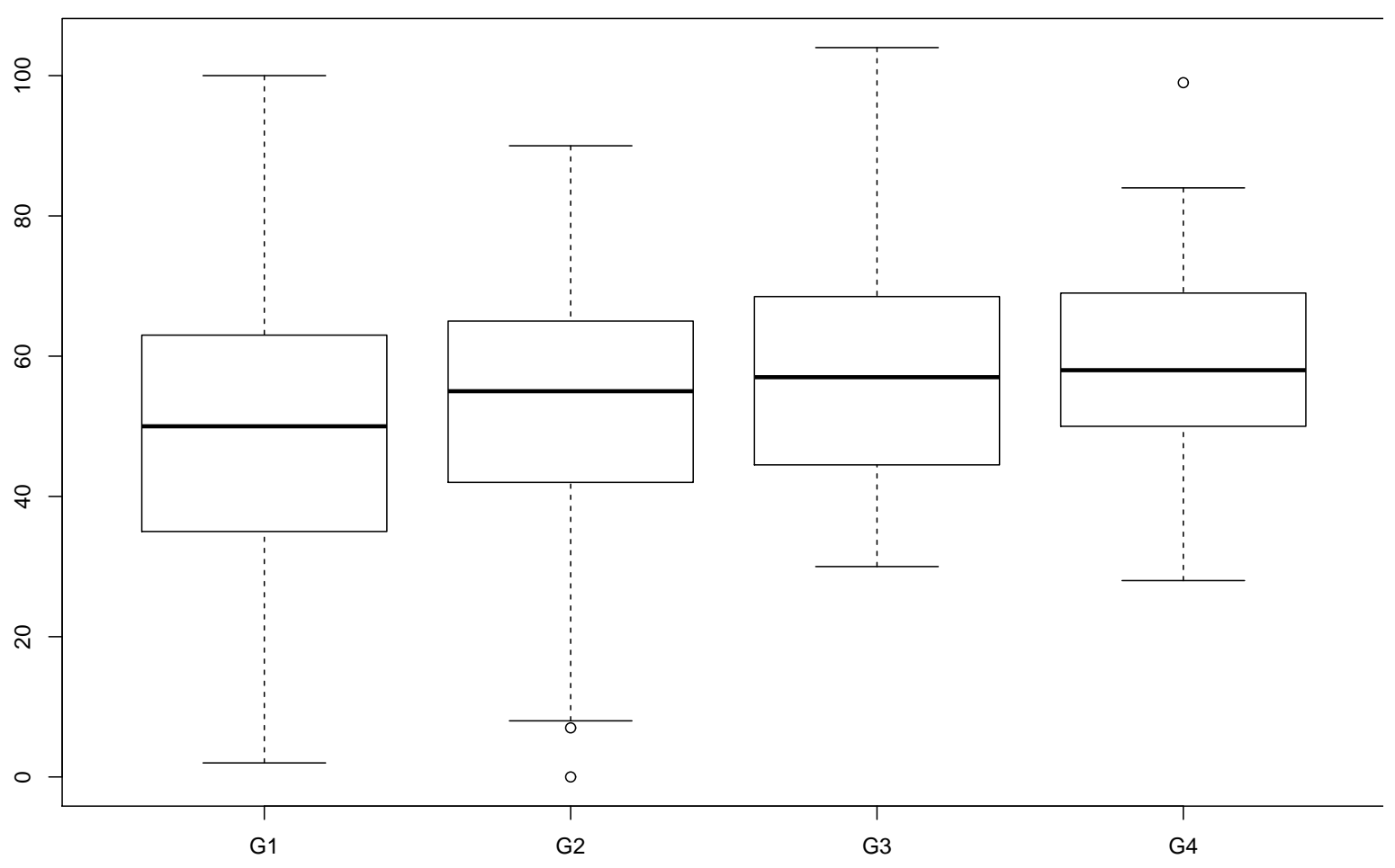

Figure 2: Boxplots for the four groups compared in the Well Elderly 2 study. G1=high school graduate, G2=some college or technical school, G3=4 years of college and G4=post-graduate school.

Figure 2 shows boxplots for each of the four groups, which suggests that more pronounced differences occur based on the lower quartile compared to upper quartile. Applying method $\mathrm{Q}$, the p-values corresponding to the $.25, .5$ and .75 quantiles were $0, .074$ and .294 , respectively. So in terms of participants who score relatively high on meaningful activities, no significant difference is found, but a significant result is found for low levels of meaningful activity as reflected by the .25 quantiles. 


\section{Concluding Remarks}

All indications are that if the cardinality of the sample space is $N>20$ and the sample size is $n \geq 30$, reasonably good control over the Type I error probability will be achieved using method Q when the goal is to compare the population medians. When comparing the quartiles, now $n \geq 50$ might be required. Of course, simulations do not guarantee that this will be the case for all practical situations that might be encountered. But the main point is that no other method has been found that performs even tolerably well in simulations when tied values are likely to occur, except for the special case of $J=2$ groups.

There are many variations of the method used here. For example, in various situations, weighted bootstrap methods have been suggested when dealing with robust estimators; see for example Salibian-Barrera and Zamar (2002) and the papers they cite. There are several alternatives to the Harrell-Davis estimator that use a weighted sum of all the order statistics, and there are variations of the projection distance that was used. perhaps there are situations where some combination of these methods provide a practical advantage over the method used here, but this remains to be determined. The main point is that the method in the paper is the only known method that continues to perform reasonably well when there are tied values.

Finally, method Q can be applied with the $\mathrm{R}$ function Qanova, which has been added to the Forge R package WRS. This function is also stored in the file Rallfun-v28, which can be downloaded from http://dornsife.usc.edu/cf/labs/wilcox/wilcox-faculty-display.cfm.

\section{REFERENCES}

Bradley, J. V. (1978) Robustness? British Journal of Mathematical and Statistical Psychology, 31, 144-152.

Bonett, D. G. \& Price, R. M. (2002). Statistical inference for a linear function of medians: Confidence intervals, hypothesis testing, and sample size requirements. Psychological Methods, \%, 370-383.

Clark, F., Azen, S. P., Zemke, R., Jackson J., Carlson, M., Mandel, D., Hay, J., Joseph- 
son, K., Cherry, B., Hessel, C., Palmer, J., \& Lipson, L . (1997). Occupational therapy for independent-living older adults. A randomized controlled trial. Journal of the American Medical Association, 278, 1321-1326.

Cliff, N. (1996). Ordinal Methods for Behavioral Data Analysis. Mahwah, NJ: Erlbaum.

Dielman, T., Lowry, C. \& Pfaffenberger, R. (1994). A comparison of quantile estimators. Communications in Statistics-Simulation and Computation, 23, 355-371.

Doksum, K. A. \& Sievers, G. L. (1976). Plotting with confidence: graphical comparisons of two populations. Biometrika, 63, 421-434.

Donoho, D. L. \& Gasko, M. (1992). Breakdown properties of the location estimates based on halfspace depth and projected outlyingness. Annals of Statistics, 20, 1803-1827.

Frigge, M., Hoaglin, D. C. \& Iglewicz, B. (1989). Some implementations of the boxplot. American Statistician, 43, 50-54.

Fung, K. Y., 1980. Small sample behaviour of some nonparametric multi-sample location tests in the presence of dispersion differences. Statistica Neerlandica, 34, 189-196.

Harrell, F. E. \& Davis, C. E. (1982). A new distribution-free quantile estimator. Biometrika, 69, 635-640.

He, X., Simpson, D. G. \& Portnoy, S. L. (1990). Breakdown robustness of tests. Journal of the American Statistical Association, 85, 446-452.

Hettmansperger, T. P. (1984). Statistical Inference Based on Ranks. New York: Wiley.

Hoaglin, D. C. (1985). Summarizing shape numerically: The g-and-h distribution. In D. Hoaglin, F. Mosteller \& J. Tukey (Eds.) Exploring Data Tables Trends and Shapes. New York: Wiley, pp. 461-515.

Hochberg, Y. (1988). A sharper Bonferroni procedure for multiple tests of significance. Biometrika, 75, 800-802.

Jackson, J., Mandel, D., Blanchard, J., Carlson, M., Cherry, B., Azen, S., Chou, C.-P., Jordan-Marsh, M., Forman, T., White, B., Granger, D., Knight, B., \& Clark, F. (2009). 
Confronting challenges in intervention research with ethnically diverse older adults:the USC Well Elderly II trial. Clinical Trials, 6 90-101.

Liu, R. G. \& Singh, K. (1997). Notions of limiting P values based on data depth and bootstrap. Journal of the American Statistical Association, 92, 266-277.

Parrish, R. S. (1990). Comparison of quantile estimators in normal sampling. Biometrics, 46, 247-257.

Pratt, J. W. (1968). A normal approximation for binomial, F, beta, and other common, related tail probabilities, I. Journal of the American Statistical Association, 63, 1457-1483.

Racine, J. \& MacKinnon, J. G. (2007). Simulation-based tests that can use any number of simulations. Communications in Statistics-Simulation and Computation, 36, 357-365.

Radloff L. (1977). The CES-D scale: a self report depression scale for research in the general population. Applied Psychological Measurement, 1, 385-401.

Romano, J. P. (1990). On the behavior of randomization tests without a group invariance assumption. Journal of the American Statistical Association, 85, 686-692.

Salibian-Barrera, M. \& Zamar, R. H. (2002). Bootstrapping robust estimates of regression. Annals of Statistics, 30, 556-582.

Sfakianakis, M. E. \& Verginis, D. G. (2006). A new family of nonparametric quantile estimators. Communications in Statistics-Simulation and Computation, 37, 337-345.

Sheather, S. J. \& Marron, J. S. (1990). Kernel quantile estimators. Journal of the American Statistical Association, 85, 410-416.

Wilcox, R. R. (2006). Comparing medians. Computational Statistics \& Data Analysis, 51, 1934-1943.

Wilcox, R. R. (2012). Introduction to Robust Estimation and Hypothesis Testing (3rd Edition). San Diego, CA: Academic Press.

Wilcox, R. R., Erceg-Hurn, D., Clark, F. \& Carlson, M. (2014). Comparing two independent groups via the lower and upper quantiles. Journal of Statistical 
Computation and Simulation, 84, 1543-1551. DOI: 10.1080/00949655.2012.754026. 